\title{
Greater Tuberosity Fractures: Does Fracture Assessment and Treatment Recommendation Vary Based on Imaging Modality?
}

\author{
Stein J. Janssen MD, Hugo H. Hermanussen BSc, Thierry G. Guitton MD, PhD, \\ Michel P. J. van den Bekerom MD, Derek F. P. van Deurzen MD, David Ring MD, PhD
}

Received: 23 September 2015/Accepted: 8 January 2016/Published online: 21 January 2016

(C) The Association of Bone and Joint Surgeons \& 2016

\begin{abstract}
Background For greater tuberosity fractures, 5-mm displacement is a commonly used threshold for recommending surgery; however, it is unclear if displacement can be assessed with this degree of precision and reliability using plain radiographs. It also is unclear if CT images provide additional information that might change decision making. Question/Purposes We asked: (1) Does interobserver agreement for assessment of the amount and direction of
\end{abstract}

One of the authors (DR) certifies that he, or a member of his immediate family, has or may receive payments or benefits, during the study period, an amount less than USD 10,000 from Wright Medical (Memphis, TN, USA); an amount less than USD 10,000 from Skeletal Dynamics (Miami, FL, USA); an amount less than USD 10,000 from Biomet (Warsaw, IN, USA); an amount less than USD 10,000 from AO North America (Paoli, PA, USA); and an amount less than USD 10,000 from AO International (Dubendorf,

Switzerland). One of the authors (HHH) certifies that he, or a member of his immediate family, has or may receive payments or benefits, during the study period, an amount less than USD 10,000 from the Anna Foundation (The Netherlands); an amount less than USD 10,000 from the Marco Polo Fund (The Netherlands); and an amount less than USD 10,000 from the Groningen University Fund (The Netherlands).

All ICMJE Conflict of Interest Forms for authors and Clinical Orthopaedics and Related Research ${ }^{\circledR}$ editors and board members are on file with the publication and can be viewed on request.

This work was performed at Massachusetts General Hospital, Boston, MA, USA.

Electronic supplementary material The online version of this article (doi:10.1007/s11999-016-4706-6) contains supplementary material, which is available to authorized users.

S. J. Janssen, H. H. Hermanussen, D. Ring ( $\square)$

Hand Service, Department of Orthopaedic Surgery,

Massachusetts General Hospital, Yawkey Center, Suite 2100, 55

Fruit Street, Boston, MA 02114, USA

e-mail: dring@partners.org fracture-fragment displacement vary based on imaging modality (radiographs only; 2-dimensional [2-D] CT images and radiographs; and 3-dimensional [3-D] and 2-D CT images and radiographs)? (2) Does the likelihood of recommending surgery vary based on imaging modality? (3) Does the level of confidence regarding the decision for treatment vary based on imaging modality?

Methods We invited 791 orthopaedic surgeons to complete a survey on greater tuberosity fractures. One hundred eighty (23\%) responded and were randomized on a 1:1:1 basis in one of the three imaging modality groups and evaluated the same set of 22 fractures. We described age, sex, mechanism of injury, days between injury and imaging, and that patients had no comorbidities or signs of neurovascular damage for every case. One hundred sixty-four of the 180 respondents completed the study and there was an imbalance in noncompletion between the three groups (two of 67 [3.0\%] in the radiograph only group; nine of 57 [16\%] in the 2-D CT and radiograph group; and five of 56 [8.9\%] in the 3-D CT, 2-D CT, and radiograph group; $p=0.043$ by Fisher's exact test). Participants assessed amount (in millimeters) and direction (posterosuperior/posteroinferior/ anterosuperior/anteroinferior/no displacement) of displacement; recommended treatment (surgical or nonoperative); and indicated their level of confidence regarding the recommended treatment on a scale from 0 to 10 for every case. Overall recommendation for treatment was expressed as a surgery score per surgeon by dividing the amount of cases they would operate on by the total number of cases $(n=22)$ and presented as a percentage. Confidence regarding the

T. G. Guitton

Department of Orthopaedic Surgery, Academic Medical Center Amsterdam, Amsterdam, The Netherlands 
decision for treatment was calculated by averaging the confidence score per surgeon, ranging from 0 to 10 . We compared interobserver agreement using kappa for categorical variables and intraclass correlation (ICC) for continuous variables. We used multivariable linear regression to assess difference in surgery score and confidence level between imaging groups, controlling for surgeon characteristics.

Results Interobserver agreement for assessment of amount (radiographs: ICC, 0.55, 2-D CT + radiographs ICC, 0.53, 3-D CT + 2-D CT + radiographs ICC, 0.57; $p$ values on all comparisons $>0.7$ ) and direction (radiographs: kappa, 0.30, 2-D CT + radiographs kappa, 0.43, 3-D CT + 2-D CT + radiographs kappa, 0.40; $p$ values for all comparisons $>0.096$ ) of displacement did not vary by imaging modality. 2-D CT and radiographs ( $\beta$ regression coefficient $[\beta], 3.1 ; p=0.253)$ and 3-D CT, 2-D CT and radiographs $(\beta, 1.6 ; p=0.561)$ did not result in a difference in recommendation for surgery compared with radiographs alone. 2-D CT and radiographs $(\beta, 0.40 ; p=$ $0.021)$ and 3-D CT, 2-D CT and radiographs $(\beta, 0.44 ; p=$ 0.011 ) were associated with slightly higher levels of confidence compared with radiographs alone.

Conclusions Imaging modality, with the numbers evaluated, does not influence interobserver agreement of greater tuberosity fracture assessment, nor did it influence the recommendation for surgical treatment. However, surgeons did feel slightly more confident about their treatment recommendation when assessing CT images with radiographs compared with radiographs alone. Our results therefore suggest no additional value of CT scans for assessment of greater tuberosity fractures when displacement seems to be minimal on plain radiographs. CT scans could be helpful in borderline cases, or in case other fractures can be expected (eg, an occult surgical neck fracture).

Level of evidence Level III, diagnostic study.

\section{Introduction}

The amount and direction of displacement that benefit from surgery in isolated greater tuberosity fractures are debatable [11, 13, 18]. Some surgeons advocate surgical treatment for as little as $3-\mathrm{mm}$ displacement, but $5 \mathrm{~mm}$ is a more-commonly used threshold for recommending surgery $[11,21]$. However, it is unclear if displacement can be assessed with this degree of precision and reliability. Some surgeons recommend CT when radiographic assessment of a greater tuberosity fracture is inconclusive [11, 13].

M. P. J. van den Bekerom, D. F. P. van Deurzen Shoulder and Elbow Unit, Department of Orthopaedic Surgery, Onze Lieve Vrouwe Gasthuis, Amsterdam, The Netherlands
Studies of fractures at other anatomic areas (such as the tibia plateau) are conflicting regarding improved fracture assessment reliability when comparing CT scans with radiographs $[5,6,22]$. Criteria used for diagnosis or treatment ideally should be reliable, accurate, and associated with outcome. The extra resources and radiation of CT scanning are merited only if $\mathrm{CT}$ provides additional information that might change decision making and improve outcomes. Advanced imaging potentially increases surgeons' level of confidence regarding the decision for treatment and increased confidence might decrease the need for second opinions, additional followup appointments, and radiographs for uncertainty.

We therefore aimed to evaluate reliability of assessment of greater tuberosity fracture characteristics and resulting treatment recommendations after different imaging modalities. Specifically, we asked the following questions: (1) Does interobserver agreement for assessment of the amount and direction of fracture-fragment displacement vary based on imaging modality? (2) Does the likelihood of recommending surgery vary based on imaging modality? (3) Does the level of confidence regarding the decision for treatment vary based on imaging modality?

\section{Materials and Methods}

\section{Study Design and Participants}

Our institutional review board approved this cross-sectional survey study. All members of the Science Of Variation Group (SOVG) were invited to complete an 88question survey on greater tuberosity fractures. The SOVG aims to study variation in assessment and treatment of upper extremity conditions, without financial incentive [4, 15]. It includes 791 orthopaedic and trauma surgeons. Of those, $180(23 \%)$ responded and participated in this study. Sixteen $(8.9 \%)$ participants did not complete the survey and were excluded, and 164 completed all questions and were kept for analysis. Because most of the members with emails in the SOVG database are not active participants, the rate of participation is not a true response rate. In addition, not all the SOVG members are shoulder or fracture specialists. Those who do not treat these injuries presumably opted out.

The survey was created online in SurveyMonkey ${ }^{\circledR}$ (Palo Alto, CA, USA). Invitations to participate were sent on July 8, 2015. At 2, 4, and 5 weeks we sent a reminder.

Participating surgeons were randomized through a builtin feature of SurveyMonkey ${ }^{\circledR}$ to one of three study arms on enrollment in the study on a 1:1:1 basis. Simple randomization was used, meaning that each new subject is assigned to a group randomly and independently of 
previous group assignments (ie, no method is used to prevent imbalance in group size [eg, permuted block randomization]) [16]. Case descriptions were the same for all three study arms, but imaging modalities varied: radiographs only (Group 1); 2-dimensional CT-scan (2-D CT) images and radiographs (Group 2); and 3-dimensional (3D) and 2-dimensional CT (3-D CT and 2-D CT) images and radiographs (Group 3) (Fig. 1).

The survey included 22 cases and each case was followed by four questions: (1) What is the estimated displacement in millimeters?; (2) What is the direction of displacement? (Posterosuperior/Posteroinferior/Anterosuperior/Anteroinferior/No displacement); (3) What treatment would you recommend? (Operative/Nonoperative); (4) On a scale from 0 to 10 , how confident are you about this decision? $(0=$ not at all confident to $10=$ very confident). The answer to Question 1 was dichotomized to less than 5 -mm displacement and $5 \mathrm{~mm}$ or more as this cutoff often is used as a threshold for recommending surgical treatment $[13,18]$. We described age, sex, mechanism of injury, and days between the injury and imaging for every case (Appendix 1. Supplemental material is available with the online version of $\mathrm{CORR}^{\circledR}$ ). We also explained that the patients had no comorbidities and that there were no signs of neurovascular damage. Sex, location of practice, years in practice, supervising trainees, and specialization of the surgeon were derived from the SOVG members' database $[4,15]$.

We used a diagnosis code (International Classification of Diseases, 9th Revision code 812.03: fracture of greater tuberosity of humerus closed) to identify patients who received the diagnosis of greater tuberosity fracture

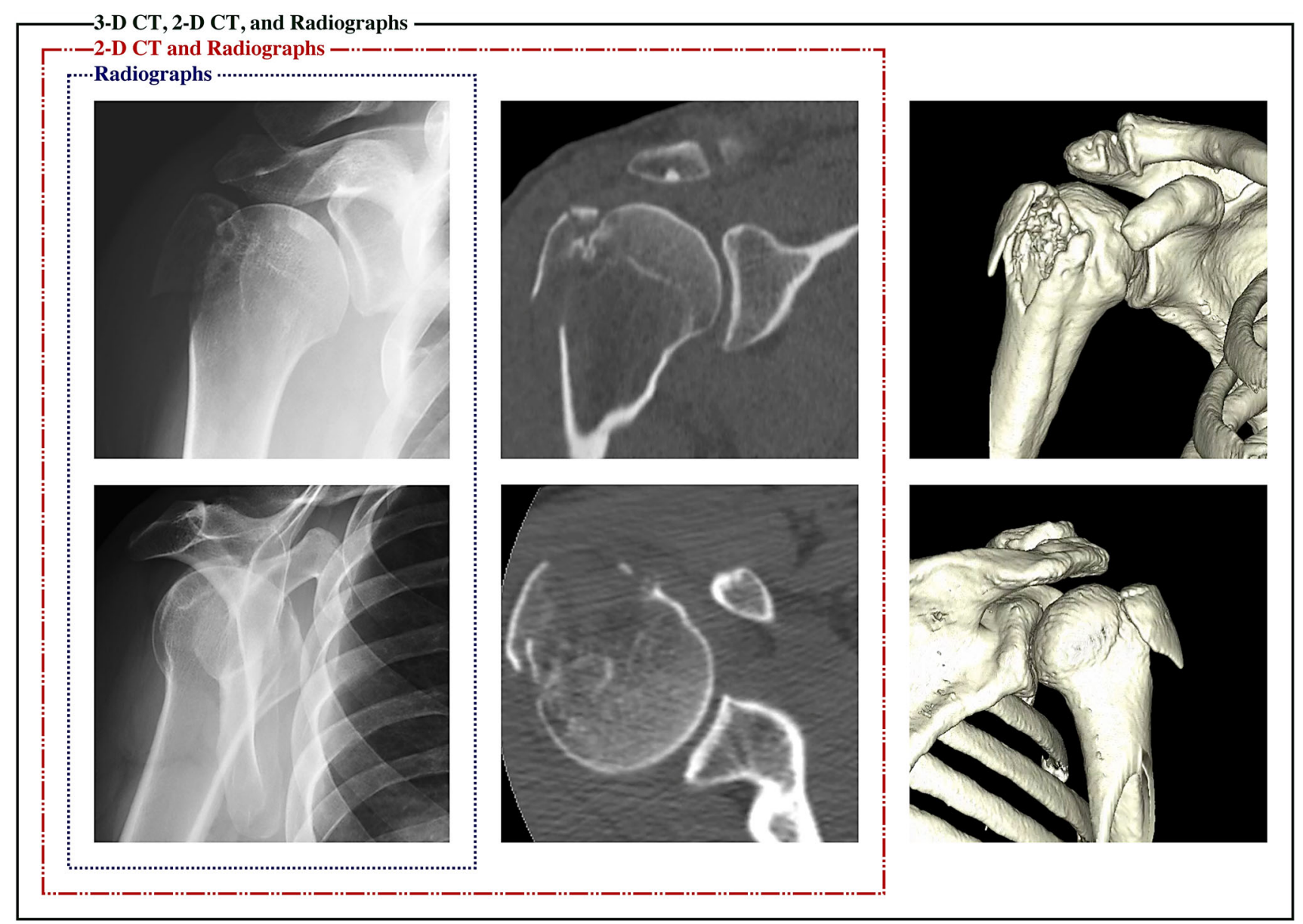

Fig. 1 A case example is shown. A 46-year-old male sustained a greater tuberosity fracture during a fall 4 days ago. The patient has no comorbidities and there are no signs of neurovascular damage. Case descriptions were the same for all three study arms, but imaging modalities varied: radiographs only (blue dotted outline); 2-dimensional CT-scan (2-D CT) images and radiographs (red dashed outline); and 3-dimensional (3-D) and 2-dimensional CT (3-D CT and 2-D CT) images and radiographs (black solid outline). Radiographs were shown as plain images, 2-D CT as scroll-through movies in the coronal and transversal planes, and 3-D CT as $180^{\circ}$ fly-through movies. 

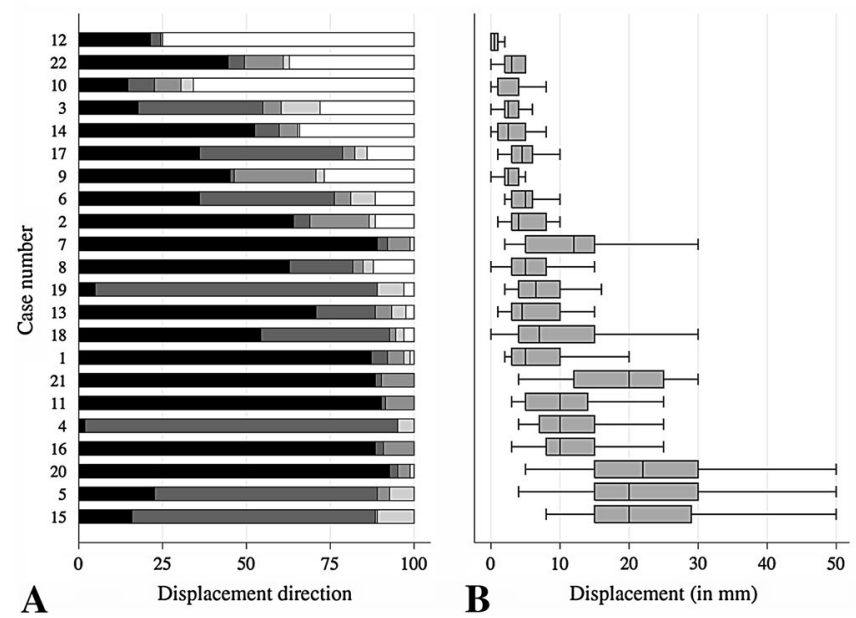

Fig. 2A-D (A) The bar graph shows the percentage of surgeons indicating posterosuperior (black bar), posteroinferior (dark gray bar), anterosuperior (medium gray bar), anteroinferior (light gray bar), and no displacement (white bar) per case. (B) The box plot shows the median amount of displacement in millimeters with interquartile range and range as estimated by the surgeons per case. The bar graphs

between January 2005 and January 2014 at one of two tertiary care clinics $(\mathrm{n}=314)$. There were $22(7.0 \%)$ isolated greater tuberosity fractures confirmed with radiographs and CT scans (Appendix 1. Supplemental material is available with the online version of $\operatorname{CORR}^{\circledR}$ ).

All available radiographs were shown for every case. Three-dimensional images were rendered from the 2-D CT scans using the OsiriX medical image viewer application (Version 6.5.2; Pixmeo, Bernex, Switzerland). Short $( \pm 10$ seconds) scroll-through movies were made, via a built-in feature of OsiriX, for the 2-D images in the coronal and transversal planes. We made $180^{\circ}$ fly-through movies of the 3-D rendered images (Fig. 1). Radiographs and CT scans were obtained for clinical purposes and were not standardized for this study. No measurement tool was available while taking the survey.

\section{Outcome Measures}

Overall recommendation for treatment was expressed as a surgery score per surgeon by dividing the amount of cases they would operate on by the total number of cases $(n=22)$ and presented as a percentage. The surgery score ranges from $0 \%$ to $100 \%$, with a higher score indicating higher likelihood for recommending surgery. Confidence regarding the decision for treatment was calculated by averaging the confidence score per surgeon; the score ranges from 0 to 10 with a higher score indicating more decisional confidence.
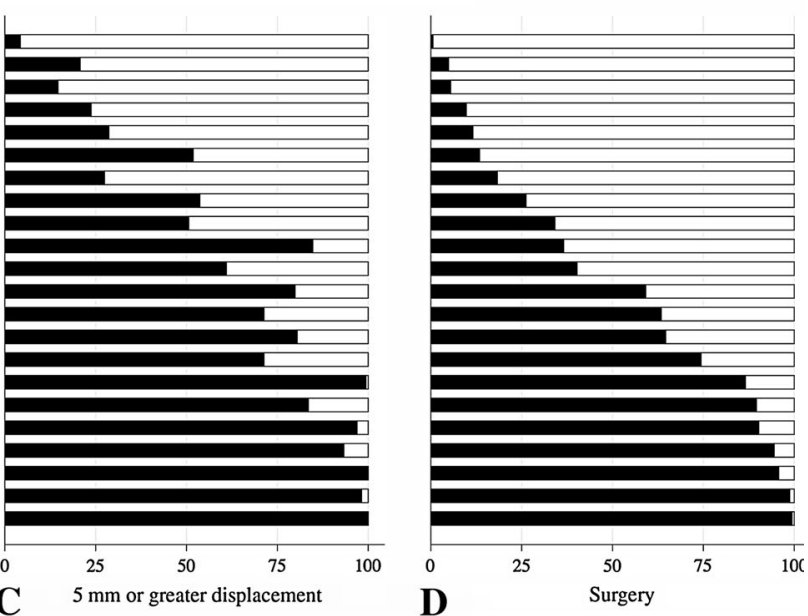

show (C) the percentage of surgeons indicating $5 \mathrm{~mm}$ or more displacement (black bar) and less than $5 \mathrm{~mm}$ (white bar), and (D) the percentage of surgeons indicating operative (black bar) and nonoperative (white bar) treatment per case. Cases are ordered from only one surgeon recommending surgery (Case 12) to all but one surgeon recommending surgery (Case 15).

\section{Surgeon Characteristics}

Sixteen of the 180 participants did not complete the questionnaire and there was an imbalance in noncompletion between the groups (two of 67 [3.0\%] in the radiograph only group; nine of 57 [16\%] in the 2-D CT and radiograph group; and five of 56 [8.9\%] in the 3-D CT, 2-D $\mathrm{CT}$, and radiograph group; $\mathrm{p}=0.043$ by Fisher's Exact test). Of the 164 participants who completed all questionnaires, $158(96 \%)$ were men. The majority of participants were from North America (46\%) and Europe (42\%). There were no differences in baseline characteristics between the three study arms (Table 1).

\section{Statistical Analysis}

Categorical variables were shown as frequencies with percentages and continuous variables as mean with SD as visual inspection of histograms suggested normality.

We screened our data for potential erroneous entries for Question 1 (estimated displacement in millimeters) as this was a free numeric field. We used Mahalanobis distance (a measure of separation between groups of values) and 1.5 times the interquartile range below the first quartile and above the third quartile to detect outliers, indicating potentially erroneous entries [9]. We replaced three values $(10-, 10-$, and 20-cm displacement of the greater tuberosity fragment) with the median value in one individual as these 
Table 1. Baseline characteristics of participating surgeons per group $(n=164)^{*}$

\begin{tabular}{|c|c|c|c|c|}
\hline Variable & $\begin{array}{l}\text { Group } 1 \\
\text { Radiographs } \\
(\mathrm{n}=65)\end{array}$ & $\begin{array}{l}\text { Group } 2 \\
2-\mathrm{D} \text { C }+ \text { radiographs } \\
(\mathrm{n}=48)\end{array}$ & $\begin{array}{l}\text { Group } 3 \\
3-\mathrm{D} C \mathrm{C}+2-\mathrm{D} \mathrm{CT}+\text { radiographs } \\
(\mathrm{n}=51)\end{array}$ & $\mathrm{p}$ value \\
\hline Sex & $\mathrm{n}(\%)$ & n $(\%)$ & n $(\%)$ & \\
\hline Males & $62(95)$ & $48(100)$ & $48(94)$ & 0.322 \\
\hline Females & $3(4.6)$ & $0(0)$ & $3(5.9)$ & \\
\hline \multicolumn{5}{|c|}{ Location of practice } \\
\hline North America & $27(42)$ & $21(44)$ & $27(53)$ & 0.182 \\
\hline Europe & $30(46)$ & $21(44)$ & $18(35)$ & \\
\hline Asia & $5(7.7)$ & $1(2.1)$ & $0(0)$ & \\
\hline Australia & $0(0)$ & $3(6.3)$ & $3(5.9)$ & \\
\hline South America & $3(4.6)$ & $2(4.2)$ & $3(5.9)$ & \\
\hline \multicolumn{5}{|l|}{ Years in practice } \\
\hline $0-5$ years & $19(29)$ & $17(35)$ & $15(29)$ & 0.501 \\
\hline $6-10$ years & $17(26)$ & $11(23)$ & $6(12)$ & \\
\hline $11-20$ years & $20(31)$ & $14(29)$ & $20(39)$ & \\
\hline $21-30$ years & $9(14)$ & $6(13)$ & $10(20)$ & \\
\hline \multicolumn{5}{|c|}{ Supervising trainees } \\
\hline Yes & $59(91)$ & $46(96)$ & $42(82)$ & 0.082 \\
\hline No & $6(9.2)$ & $2(4.2)$ & $9(18)$ & \\
\hline \multicolumn{5}{|l|}{ Specialization } \\
\hline Hand-wrist & $19(29)$ & $8(17)$ & $12(24)$ & 0.268 \\
\hline Traumatology & $23(35)$ & $23(48)$ & $19(37)$ & \\
\hline Shoulder-elbow & $12(18)$ & $14(29)$ & $15(29)$ & \\
\hline Other & 11 (17) & $3(6.3)$ & $5(10)$ & \\
\hline
\end{tabular}

* Randomized to one of the three groups by SurveyMonkey ${ }^{\mathbb{R}}$

seemed erroneous; these values were more than twice as great as the second-largest value.

We assessed interobserver agreement in assessment of amount and direction of displacement, and recommendation for surgery per imaging modality group. We used Fleiss kappa analysis to assess the interobserver agreement for the categorical variables direction of displacement, 5$\mathrm{mm}$ displacement, and recommendation for surgery. Kappa is a quantitative measure of agreement accounting for agreement by chance among observers and ranges from 0 to 1. A kappa value of 1 reflects perfect agreement, whereas a kappa of 0 indicates agreement based on chance. According to Landis and Koch [17], a kappa between 0.01 and 0.20 is considered to reflect slight agreement, a value between 0.21 and 0.40 as fair agreement, between 0.41 to 0.60 as moderate agreement, 0.61 and 0.80 as substantial agreement, and greater than 0.81 as almost perfect agreement.

Bootstrapping (number of resamples: 1000) was used to calculate the standard error, $\mathrm{z}$-statistic, $95 \% \mathrm{CIs}$, and $\mathrm{p}$ values for the kappa values to compare groups. We used an intraclass correlation coefficient (ICC) through a two-way mixed-effects model with absolute agreement to assess difference in agreement for the continuous variable displacement. Absolute agreement in an ICC assesses how much each measurement performed per observer differs from the other observers. A score of 1 indicates perfect agreement, whereas 0 reflects no agreement. Fisher's Z transformation was used to calculate $\mathrm{p}$ values comparing individual groups [1,3].

Multivariable linear regression analysis was used to assess difference in surgery score and confidence level between groups, controlling for possible imbalance in baseline surgeon characteristics of sex, location of practice (Asia, Australia, and South America were grouped as other owing to their small size), years in practice, supervising trainees, and specialization. The $\beta$ regression coefficients, derived from linear regression, indicate the absolute difference in outcome (surgery score and confidence level) in one group compared with a reference group.

A sample size calculation for difference in interobserver agreement between groups showed that we were able to detect a medium effect size of 0.54 with a sample size of 164 participants, assuming equal distribution of participants among the three groups, and $80 \%$ statistical power $(\beta=$ $0.20 ; \alpha=0.05)$. A second sample-size calculation for 
difference in surgery score and confidence level among imaging groups showed that we were able to detect a small effect size of 0.24 with a sample size of 164 participants, assuming equal distribution of participants among the three groups, and $80 \%$ statistical power $(\beta=0.20 ; \alpha=0.05)$ using one-way ANOVA.

A two-tailed $\mathrm{p}$ value less than 0.05 was considered significant; all statistical analyses were performed using Stata ${ }^{\circledR} 14$ (StataCorp LP, College Station, TX, USA). There were no missing values for any of the variables.

\section{Results}

\section{Interobserver Agreement}

We found no difference, with the numbers available, in interobserver agreement for assessment of displacement in millimeters, direction of displacement, and the 5-mm cutoff between the radiographs only, 2-D CT and radiographs, and 3-D CT, 2-D CT, and radiographs groups.

The ICC was 0.55 (95\% CI, 0.41-0.71) for estimating the amount of displacement on radiographs only, 0.53 (95\% CI, $0.39-0.70$ ) for the 2-D CT and radiographs group, and 0.57 (95\% CI, 0.43-0.74) for the 3-D CT, 2-D CT, and radiographs group; these did not differ between groups (radiographs versus 2-D CT and radiographs, $\mathrm{p}=0.913$; radiographs versus 3-D CT, 2-D CT, and radiographs, $\mathrm{p}=$ 0.836; and 2-D CT and radiographs versus 3-D CT, 2-D CT, and radiographs, $\mathrm{p}=0.769)$. The kappa was $0.30(95 \% \mathrm{CI}$, $0.20-0.40$ ) for assessing direction of displacement on radiographs only, 0.43 (95\% CI, 0.31-0.55) for the 2-D CT and radiographs group, and $0.40(95 \% \mathrm{CI}, 0.29-0.50)$ for the 3-D CT, 2-D CT, and radiographs group; these did not differ between groups (radiographs versus 2-D CT and radiographs, $\mathrm{p}=0.096$; radiographs versus 3-D CT, 2-D CT, and radiographs, $\mathrm{p}=0.182$; and 2-D CT and radiographs versus 3-D CT, 2-D CT, and radiographs, $\mathrm{p}=0.686$ ). The kappa was 0.46 (95\% CI, 0.31-0.60) for establishing the 5-mm cut-off on radiographs only, 0.45 (95\% CI, 0.30-0.60) for the 2-D $\mathrm{CT}$ and radiographs group, and 0.40 (95\% CI, 0.25-0.55) for the 3-D CT, 2-D CT, and radiographs group; these did not differ between groups (radiographs versus 2-D CT and radiographs, $p=0.964$; radiographs versus 3-D CT, 2-D CT, and radiographs, $\mathrm{p}=0.587$; and 2-D CT and radiographs versus 3-D CT, 2-D CT, and radiographs, $\mathrm{p}=0.626$ ).

\section{Recommendation for Treatment}

After controlling for relevant confounding variables (sex, location of practice, years in practice, supervising trainees, and specialization), 2-D CT and radiographs ( $\beta$ regression coefficient $[\beta]$ 3.1; standard error $[\mathrm{SE}] 2.7 ; 95 \% \mathrm{CI},-2.3$ to $8.6 ; \mathrm{p}=0.253$ ) and 3-D CT, 2-D CT, and radiographs ( $\beta$ 1.6; SE, $2.7 ; 95 \% \mathrm{CI},-3.8$ to $6.9 ; \mathrm{p}=0.561$ ) did not result in a difference in recommendation for surgery (ie, surgery score) compared with radiographs alone. The surgery score was $49 \%$ (SD, 13\%) for radiographs only; $53 \%$ (SD, 14\%) for 2-D CT and radiographs; and 52\% (SD, 15\%) for 3-D CT, 2-D CT, and radiographs.

None of the other explanatory variables (sex, location of practice, years in practice, supervising trainees, specialization of the surgeon) was independently associated with a higher surgery score.

\section{Confidence in Decision-making}

After controlling for relevant confounding variables (sex, location of practice, years in practice, supervising trainees, and specialization), 2-D CT and radiographs ( $\beta, 0.40 ; \mathrm{SE}$, 0.17 ; 95\% CI, 0.062-0.75; $\mathrm{p}=0.021)$ and 3-D CT, 2-D CT, and radiographs $(\beta, 0.44 ; \mathrm{SE}, 0.17 ; 95 \% \mathrm{CI}, 0.10-0.78 ; \mathrm{p}=$ 0.011 ) were associated with slightly higher levels of confidence compared with radiographs alone. It is questionable whether this small difference is clinically important. The confidence level was 7.6 (SD, 0.90) for radiographs only; 8.0 (SD, 0.89) for 2-D CT and radiographs; and 8.1 (SD, 0.85 ) for 3-D CT, 2-D CT, and radiographs.

None of the other explanatory variables (sex, location of practice, years in practice, supervising trainees, specialization of the surgeon) was independently associated with a higher confidence level.

\section{Additional Finding}

We found a strong association of displacement with surgery score $(\beta, 4.6$; SE, 0.66; 95\% CI, 3.3-6.0; $p<0.001$; adjusted R-squared, 0.70) when averaging displacement and surgery score for the 22 cases. This confirms that displacement drives the decision for surgery in patients with greater tuberosity fractures (Fig. 2).

\section{Discussion}

Displacement of a greater tuberosity fracture is used to decide on surgery, but it is not clear if its assessment on radiographs is reliable, and if more advanced imaging (CT scanning) provides additional information that might change decision making. We found that 2-D or 3-D CT images, in addition to radiographs, with the numbers available, do not influence interobserver agreement of greater tuberosity fracture assessment, nor did the addition 
of these images influence the recommendation for surgical treatment. However, surgeons did feel slightly more confident about their treatment recommendation when assessing CT images with radiographs compared with radiographs alone.

This study has limitations. First, only $23 \%$ of the surgeons in the SOVG responded to the questionnaire and surgeons in the SOVG are only a subgroup; most of them are in academic medicine (80\% [631/791] supervises trainees) and their values, training, and practice probably differ from those of the larger community of orthopaedic surgeons. We found a difference between responding and nonresponding SOVG members for location of practice (respondents: Europe: 40\%; North America: 48\%; and other: $12 \%$, versus nonrespondents: Europe: $25 \%$; North America: 62\%; and other: 13\%; p < 0.001, by Fisher's exact test), involvement in academic medicine (respondents: $89 \%$ and nonrespondents: 77\%; p $<0.001$, by Fisher's exact test), and their primary specialization (respondents: trauma: 42\%; shoulder-elbow: 25\%; handwrist: $24 \%$; and general orthopaedics: $8 \%$, versus nonrespondents: trauma: 29\%; shoulder-elbow: 14\%; handwrist: $43 \%$, and general orthopaedics: $15 \%)$. Sex ( $\mathrm{p}=$ 0.098 , by Fisher's exact test), and years in practice ( $\mathrm{p}=$ 0.77 , by Fisher's exact test) did not differ between responding and nonresponding SOVG members. However, we do not believe that the difference between responding and nonresponding members, or the SOVG being a subgroup of the larger community of orthopaedic surgeons, compromises the generalizability of our results as none of the surgeon characteristics (sex, location of practice, years in practice, supervising trainees, or specialization) was independently associated with the recommendation for treatment or decisional confidence. We therefore believe that our results are generalizable to the community of surgeons who treat greater tuberosity fractures. Second, the level of experience in treating greater tuberosity fractures probably differed between participating surgeons; however, we accounted for this by randomizing surgeons into groups, thereby eliminating the risk of confounding, and adjusted for all explanatory variables in multivariable analyses. We therefore see this as a minor limitation.

Third, we included only patients who had a radiograph and a CT scan of the shoulder and our sample therefore might be different than the overall patient population with greater tuberosity fractures. Patients in our study were likely selected for CT owing to more severe or complex fracture configurations. However, a wide variety of fracture patterns was included in our study (Fig. 2). Nevertheless, inclusion of a different subset of cases (eg, only "borderline" cases in which the displacement is close to the $5-\mathrm{mm}$ threshold for surgery) might have resulted in different findings and could have shown that CT imaging is useful in these specific cases. We therefore see this as an important limitation of our study. Fourth, with our sample size we found no difference in interobserver agreement of fracture characteristic assessment or recommendation for surgery among imaging modality groups; however, a larger sample size might have identified a difference. We see the relatively small sample size as a minor limitation; inclusion of more observers might have detected a difference, albeit small, and its relevance would be questionable. Fifth, we used plain images and movies instead of a Digital Imaging and Communications in Medicine (DICOM) viewer; this precludes the use of measurement tools and adjustment of contrast in our study. This might have influenced the interobserver agreement overall; however, we believe that this weakness applied to all groups and did not compromise our comparison of reliability among groups, therefore we see this as a minor limitation. Sixth, the noncompletion rate of observers differed among groups and was highest in the 2-D CT and radiographs group. We see this as a minor limitation as comparison of baseline characteristics among those who completed the study did not suggest imbalance (Table 1) and we adjusted for all baseline characteristics in multivariable analyses. Seventh, we did not assess interobserver agreement for assessment of degree of comminution. This could be an important factor for surgical decision-making. We see this as a minor limitation as it does not influence our current analyses.

Our study showed that, with the numbers available, there was no influence of imaging modality on reliability of greater tuberosity fracture characterization. Previous studies of other anatomic areas are conflicting when comparing interobserver agreement among imaging modalities [5, 22]. For example, Brunner et al. [5] found higher reliability of tibia plateau fracture classification on CT images with radiographs compared with radiographs alone, whereas te Stroet et al. [22] did not find a difference in reliability for classification and treatment recommendation between CT images and radiographs for these fractures. This discrepancy might be explained by the relatively low number of observers, inclusion of different fracture subtypes, and difference in quality of the imaging. The strengths of our study are the large number of observers with varying levels of experience and inclusion of a varied patient sample. We therefore believe that our findings are valid and generalizable to patients with greater tuberosity fractures and that there is no additional value of CT scanning in the majority of these cases. One also should consider the extra resources and radiation of $\mathrm{CT}$ scanning: the mean radiation dose for a CT scan of the shoulder $(2.1 \mathrm{mSv})$ corresponds to approximately 26 conventional posteroanterior chest radiographs or plain shoulder radiographs (both $0.08 \mathrm{mSv}$ ) $[2,12,20]$ and the costs for a shoulder CT scan (unadjusted 
Medicare fee \$180) is approximately six times the costs of plain shoulder radiographs (unadjusted Medicare fee \$29) [8]. The additional value of CT imaging might be different for other fractures and our results therefore are not generalizable to other anatomic locations [14].

Although our study showed that additional CT imaging slightly increased surgeons' confidence in making a recommendation for treatment, we found no evidence that this led to a change in recommending treatment. However, the difference in confidence score was only 0.5 point on a 10point scale and therefore unlikely to be clinically important. This is in line with a study of 145 patients with low back pain who were randomly assigned to "no imaging" and "imaging" groups. There was no difference in diagnosis or treatment plan between groups, but the clinicians' diagnostic and treatment confidence were greater in the "imaging" group compared with the group that did not have imaging [10]. More advanced CT-based measuring methods, such as quantitative 3-D CT analysis, might improve fracture assessment and result in greater agreement when recommending treatment [19]. We found it interesting that the $\mathrm{CT}$ scans did not result in different treatment recommendation, as advanced imaging often results in "case finding".

Our additional analysis showed a strong association of amount of displacement with recommendation for surgery, suggesting that this factor drives surgical decision-making. This is in line with previous studies emphasizing the importance of displacement [7, 11, 13, 18, 21].

We found no difference, with the numbers evaluated, in interobserver agreement for greater tuberosity fracture characteristic assessment, amount and direction of displacement, between imaging modalities. Imaging modality did not influence the recommendation for surgical treatment; however, surgeons did feel slightly more confident regarding their treatment recommendation when assessing CT images with radiographs compared with radiographs alone. Our results therefore suggest no additional value of CT scans for assessment of greater tuberosity fractures when displacement seems to be minimal on plain radiographs. CT scans could be helpful in borderline cases, or in case other fractures can be expected (eg, an occult surgical neck fracture).

Acknowledgments We thank the members of the Science of Variation Group: Jeffrey Abrams; Lars E. Adolfsson; Ngozi M. Akabudike; Michael H. Amini; Platz Andreas; Luke S. Austin; George C. Babis; Efstathios G. Ballas; H. Brent Bamberger; Antonio Barquet; Camilo Jose Romero Barreto; Fred Baumgaertel; Taizoon Baxamusa; Grant J. Bayne; Ramon de Bedout; Thierry Begue; Jan Biert; Julius Bishop; Lars C. Borris; Jonathan Braman; Drago Brilej; Henry Broekhuyse; Richard Buckley; John T. Capo; Aakash Chauhan; Pradeep Choudhari; Joseph M. Conflitti; Ralph M. Costanzo; Brian J. Cross; Phani Dantuluri; Thomas DeCoster; Gregory DeSilva; Thomas Dienstknecht; Filip Celestyn Dolatowski; Job N. Doornberg;
Scott F. Duncan; Holger Durchholz; George S.M. Dyer; Percy V. van Eerten; Carl Ekholm; Nelson Elias; Peter J. Evans; Denise Eygendaal; Jason C. Fanuele; Naquira Escobar Luis Felipe; Carlos H. Fernandes; Jose Eduardo Grandi Ribeiro Filho; Thomas J. Fischer; Jochen Fischer; Frede Frihagen; Simon Frostick; Guillaume Gadbled; Christos Garnavos; Grant Garrigues; Richard S. Gilbert; James A. Gillespie; Taco Gosens; J. Carel Goslings; Michael W. Grafe; Lob Guenter; Roger G. Hackney; Ian Harris; Robert Haverlag; Bernard F. Hearon; Huub van der Heide; Sven H. van Helden; Eric Hofmeister; Prashanth Inna; John Itamura; Peter Jebson; Richard Jenkinson; Kyle Jeray; Axel Jubel; Scott G. Kaar; Karoush Kabir; Nikolaos K. Kanakaris; Matej Kastelec; Leonid Katolik; Stephen A. Kennedy; Michael W. Kessler; Cyrus Klostermann; Georges Kohut; George Kontakis; Gerald A. Kraan; Peter Krause; Anze Kristan; Paul Levin; Ofer Levy; Panagiotis Lygdas; Chad Manke; Lawrence Marsh; Paul A. Martineau; Iain McGraw; Mike Mckee; Eitan Melamed; Milind Merchant; Ladislav Mica; Thomas Mittlmeier; Constanza L. Moreno-Serrano; Jesus Moreta-Suarez; Matt Mormino; Joel Murachovsky; Michael Nancollas; Valentin Neuhaus; Vasileios S. Nikolaou; Betsy M. Nolan; Timothy Omara; Reza Omid; Richard S. Page; Paolo Paladini; M. Jason Palmer; Nata Parnes; Minoo Patel; Juan M. Patiño; Emilija Stojkovska Pemovska; Rodrigo Pesantez; Anil Peters; Steve Petersen; Vial Philippe; Miguel A. Pirela-Cruz; Marinis Pirpiris; Lodewijk M.S.J. Poelhekke; Daniel Polatsch; Giuseppe Porcellini; Michael Prayson; Marc J. Richard; Martin Richardson; Marco Rizzo; Edward K. Rodriguez; Juan Miguel Rodríguez Roiz; Nigel Rossiter; Sergio Rowinski; Jorge Rubio; Parag Sancheti; Michiel A.J. van de Sande; Wojciech Satora; Peter Schandelmaier; Johan H. Scheer; Tim Schepers; Benjamin W. Sears; Mohamed Shafi; Adam B. Shafritz; Nicholas L. Shortt; Todd Siff; Raymond Malcolm Smith; Catherine Spath; Fabio Suarez; Marc Swiontkowski; David E. Tate; Eric T. Tolo; Minos Tyllianakis; Thomas F. Varecka; Michael Verhofstad; Anne J.H. Vochteloo; Robert Wagenmakers; Richard Wallensten; Frank L. Walter; Daniel C. Wascher; Mohammed Waseem; Lawrence Weiss; Brian P.D. Wills; and A.L. van der Zwan.

\section{References}

1. Bernstein ML, Chung KC. Hand fractures and their management: an international view. Injury. 2006;37:1043-1048.

2. Biswas D, Bible JE, Bohan M, Simpson AK, Whang PG, Grauer JN. Radiation exposure from musculoskeletal computerized tomographic scans. J Bone Joint Surg Am. 2009;91:1882-1889.

3. Bond C Jr, Richardson K. Seeing the FisherZ-transformation. Psychometrika. 2004;69:291-303.

4. Bruinsma WE, Guitton TG, Warner JJ, Ring D; Science of Variation Group. Interobserver reliability of classification and characterization of proximal humeral fractures: a comparison of two and three-dimensional CT. J Bone Joint Surg Am. 2013;95:1600-1604.

5. Brunner A, Horisberger M, Ulmar B, Hoffmann A, Babst R. Classification systems for tibial plateau fractures; does computed tomography scanning improve their reliability? Injury. 2010;41:173-178

6. Chan PS, Klimkiewicz JJ, Luchetti WT, Esterhai JL, Kneeland JB, Dalinka MK, Heppenstall RB. Impact of CT scan on treatment plan and fracture classification of tibial plateau fractures. $J$ Orthop Trauma. 1997;11:484-489.

7. Chun JM, Groh GI, Rockwood CA Jr. Two-part fractures of the proximal humerus. J Shoulder Elbow Surg. 1994;3:273-287.

8. CodeMap ${ }^{\mathbb{R}}$. Available at: https://www.codemap.com. Accessed December 21, 2015.

9. Finch WH. Distribution of variables by method of outlier detection. Front Psychol. 2012;3:211. 
10. Gillan MG, Gilbert FJ, Andrew JE, Grant AM, Wardlaw D, Valentine NW, Gregori AC; Scottish Back Trial Group. Influence of imaging on clinical decision making in the treatment of lower back pain. Radiology. 2001;220:393-399.

11. Green A, Izzi J Jr. Isolated fractures of the greater tuberosity of the proximal humerus. J Shoulder Elbow Surg. 2003;12:641-649.

12. Gregory T, Hansen U, Khanna M, Mutchler C, Urien S, Amis AA, Augereau B, Emery R. A CT scan protocol for the detection of radiographic loosening of the glenoid component after total shoulder arthroplasty. Acta Orthop. 2014;85:91-96.

13. Gruson KI, Ruchelsman DE, Tejwani NC. Isolated tuberosity fractures of the proximal humeral: current concepts. Injury. 2008;39:284-298.

14. Harness NG, Ring D, Zurakowski D, Harris GJ, Jupiter JB. The influence of three-dimensional computed tomography reconstructions on the characterization and treatment of distal radial fractures. J Bone Joint Surg Am. 2006;88:1315-1323.

15. Janssen SJ, Molleman J, Guitton TG, Ring D. What middle phalanx base fracture characteristics are most reliable and useful for surgical decision-making? Clin Orthop Relat Res. 2015;473:3943-3950.

16. Kim J, Shin W. How to do random allocation (randomization). Clin Orthop Surg. 2014;6:103-109.
17. Landis JR, Koch GG. The measurement of observer agreement for categorical data. Biometrics. 1977;33:159-174.

18. Mattyasovszky SG, Burkhart KJ, Ahlers C, Proschek D, Dietz SO, Becker I, Muller-Haberstock S, Muller LP, Rommens PM. Isolated fractures of the greater tuberosity of the proximal humerus: a long-term retrospective study of 30 patients. Acta Orthop. 2011;82:714-720.

19. Mellema JJ, Janssen SJ, Guitton TG, Ring D. Quantitative 3dimensional computed tomography measurements of coronoid fractures. J Hand Surg Am. 2015;40:526-533.

20. Mettler FA J., Wiest PW, Locken JA, Kelsey CA. CT scanning: patterns of use and dose. J Radiol Prot. 2000;20:353-359.

21. Park TS, Choi IY, Kim YH, Park MR, Shon JH, Kim SI. A new suggestion for the treatment of minimally displaced fractures of the greater tuberosity of the proximal humerus. Bull Hosp Jt Dis. 1997;56:171-176.

22. te Stroet MA, Holla M, Biert J, van Kampen A. The value of a CT scan compared to plain radiographs for the classification and treatment plan in tibial plateau fractures. Emerg Radiol. 2011;18:279-283. 